\title{
Polymorphisms of the TAP2 transporter gene in systemic lupus erythematosus
}

\author{
E J Davies, R P Donn, M C Hillarby, D M Grennan, W E R Ollier
}

\begin{abstract}
Objectives-To determine whether the TAP2 transporter gene, which lies between HLA-DP and HLA-DQ, is involved in determining susceptibility to systemic lupus erythematosus (SLE).

Methods-TAP2 types were determined by ARMS-PCR in 89 white patients with SLE and 156 control subjects.

Results-No particular TAP2 dimorphism or allele was associated with SLE or with any clinical/immunological subgroup of SLE. Furthermore, there was no evidence for significant linkage disequilibrium between TAP2 and HLA-DQ/DR in SLE.

Conclusions-These data suggest that TAP2 is not a disease susceptibility gene for SLE and that the disease-predisposing haplotypes do not extend as far as TAP2. This indicates that any HLADP association with SLE must be independent of other class II (DQ/DR) associations.
\end{abstract}

(Ann Rheum Dis 1994; 53: 61-63)

Systemic lupus erythematosus (SLE) is a clinically heterogeneous connective tissue disorder characterised by immunological abnormalities including autoantibodies to double-stranded (ds) and single-stranded (ss) DNA and to various soluble cellular antigens, such as Ro, La, U1-RNP and Sm. The role of these autoantibodies in the aetiopathogenesis of SLE is poorly understood.

There is a genetic predisposition to the

University of Manchester

Rheumatic Diseases Centre, Hope Hospital, Salford M6 8HD

E J Davies

M C Hillarby

ARC Epidemiology

Research Unit,

Stopford Building,

University of

Manchester, Oxford

Road, Manchester

M13 9PT

R P Donn

W E R Ollier

Department of Rheumatology, Royal North Shore Hospital, St Leonards, NSW 2065, Australia D M Grennan Correspondence to: DrEJ Davies

Accepted for publication 13 October 1993 disease demonstrated by a concordance of approximately $24 \%$ in monozygotic twins ${ }^{1}$ compared with only $3 \%$ in dizygotic twins. ${ }^{2}$ Part of this genetic predisposition is accounted for by genes within the MHC. Several recent studies have suggested that HLA-DQ genes (rather than DR or complement C4) may determine susceptibility to SLE..$^{3-5}$

The TAP2 (Transporter associated with Antigen Processing 2) gene lies between HLA$\mathrm{DP}$ and DQ in the class II region and is a member of the ABC superfamily of transporter genes. ${ }^{67}$ Together with TAP1, it forms a complex involved in the transport of endogenous peptides into the endoplasmic reticulum for presentation by MHC class I antigens. ${ }^{8}$ Both TAP1 and TAP2 genes are polymorphic (with TAP2 being the more polymorphic) and represent candidate disease susceptibility genes for SLE.
In the present study we have determined the frequences of TAP2 dimorphisms in our SLE patient population and control groups, to determine if TAP2 is involved in genetic predisposition to SLE.

\section{Materials and methods}

SLE AND CONTROL POPULATIONS

The patient population used in the study consisted of 92 white SLE patients, as previously described. ${ }^{5}$ All patients fulfilled the 1982 ARA revised criteria for SLE. ${ }^{9}$ The control population comprised 156 healthy volunteers.

DNA EXTRACTION

Genomic DNA was extracted from peripheral blood leucocytes as described previously. ${ }^{5}$

TAP2 TYPING

Each TAP2 dimorphism (isoleucine or valine at amino acid (aa) position 379 , alanine or threonine at aa positions 565 and 665) was investigated using ARMS-PCR, as previously described. ${ }^{10}$ TAP2 alleles were assigned by the haplotype associations of these dimorphic variants: $\mathrm{TAP} 2 \mathrm{~A}=\mathrm{V}-379 \quad \mathrm{~A}-565 \quad \mathrm{~T}-665$, TAP2B $=$ V-379 A-565 A-665, TAP2C = I379 A-565 T-665, TAP2D $=\mathrm{I}-379$ T-565 $\mathrm{T}-665$ and TAP2E $=\mathrm{V}-379 \mathrm{~T}-565 \mathrm{~T}-665$.

DETECTION OF AUTOANTIBODIES

Detection and quantification of antibodies to double-stranded DNA was carried out using a radioimmunoassay kit (Amesham Int). The presence of autoantibodies to four soluble cellular antigens (Ro, La, RNP, Sm) was determined by immunodiffusion and counterimmunoelectrophoresis.

STATISTICAL METHODS

The $\chi^{2} 2 \times 2$ analysis (with Yates' correction) was used to determine the significance of a frequency difference between two groups (significance level $5 \%$ ). The $\mathrm{p}$ values obtained were corrected for the number of comparisons made (that is, depending upon the number of variants and the number of groups tested).

When analysing linkage disequilibrium between TAP2 and other loci, delta values $(\Delta)$ were calculated. $\chi^{2}$ analysis was used to determine the significance of linkage disequilibrium. 
Table 1 TAP2 genotype frequencies (\%) in SLE and control populations

\begin{tabular}{lrrrrrrrrr}
\hline aa Position & $V V$ & \multicolumn{1}{c}{$\begin{array}{r}\text { VI } \\
\end{array}$} & \multicolumn{1}{c}{$I I$} & $A A$ & \multicolumn{1}{c}{ AT } & $T T$ & $A A$ & $A T$ & $T T$ \\
\hline SLE (n= 89) & 79 & 15 & 7 & 83 & 17 & 0 & 3 & 38 & 58 \\
DNA (n=57) & 79 & 14 & 7 & 86 & 14 & 0 & 2 & 42 & 56 \\
La (n=16) & 88 & 0 & 13 & 75 & 25 & 0 & 0 & 31 & 69 \\
Ro (n=12) & 58 & 25 & 17 & 92 & 8 & 0 & 0 & 33 & 67 \\
RNP (n=15) & 60 & 33 & 7 & 60 & 40 & 0 & 7 & 27 & 67 \\
Renal (n=23) & 83 & 9 & 9 & 87 & 13 & 0 & 0 & 43 & 57 \\
Vasc (n=10) & 100 & 0 & 0 & 90 & 10 & 0 & 0 & 50 & 50 \\
Controls (n= 156) & 81 & 15 & 4 & 85 & 15 & 1 & 5 & 40 & 54 \\
\hline
\end{tabular}

Abbreviations used in tables 1 and 2 are as follows: DNA = circulating antibodies to dsDNA $\mathrm{La}=$ circulating antibodies to both $\mathrm{Ro}$ and $\mathrm{La}$; $\mathrm{Ro}=$ circulating antibodies to Ro alone; RNP = circulating antibodies to U1 RNP; Renal = renal disease; Vasc = vasculitis

Table 2 TAP2 allele frequencies (\%) in SLE and control populations

\begin{tabular}{llllrr}
\hline Allele & $A$ & $B$ & $C$ & \multicolumn{1}{c}{$D$} & \multicolumn{1}{c}{$E$} \\
\hline SLE (n= 89) & 82 & 39 & 15 & 11 & 4 \\
DNA (n=57) & 81 & 44 & 16 & 9 & 5 \\
La (n=16) & 88 & 31 & 13 & 13 & 13 \\
Ro (n=12) & 75 & 33 & 42 & 8 & 0 \\
RNP (n=15) & 67 & 33 & 13 & 33 & 7 \\
Renal (n=23) & 87 & 44 & 13 & 9 & 4 \\
Vasc (n=10) & 90 & 50 & 0 & 0 & 10 \\
Controls (n=156) & 81 & 45 & 12 & 10 & 6 \\
\hline
\end{tabular}

\section{Results}

The frequencies of TAP2 dimorphisms in patients with SLE and controls are shown in table 1. Results could not be obtained for three of the 92 patients with SLE. No TAP2 dimorphism was found to be significantly associated with SLE or with any clinical or immunological subgroup of SLE. Patients with anti-U1 RNP antibodies were, however, more likely to possess threonine at amino acid position $565(\mathrm{p}=0.02$, corrected $\mathrm{p}=$ not significant).

Table 2 illustrates TAP2 allele frequencies in SLE and controls. Again, no associations with SLE were observed. TAP2 allele frequencies were also determined in various clinical and immunological subgroups of SLE. TAP2C frequency was increased in patients with circulating anti-Ro (but no anti-La) antibodies ( $p=0 \cdot 02$, corrected $p=$ not significant $)$, while TAP2D frequency was increased in patients with anti-U1 RNP antibodies $(p=0.01$, corrected $p=$ not significant). No associations were seen in patients with circulating autoantibodies to $\mathrm{La}$ or dsDNA, or in patients with renal disease or vasculitis.

We have also analysed our data to determine linkage disequilibria between TAP2 and HLADQ/DR in SLE patients. TAP2B was positively associated with DR1 $(\Delta=0.025$, $\mathrm{p}=0.05)$ and negatively associated with DR3 $(\Delta=-0.062, \quad p=0.04)$. These associations were not statistically significant upon correcting the $\mathrm{p}$ values. No other linkage disequilibria were observed.

\section{Discussions}

The TAP1 and TAP2 genes are potentially important in immune responses due to their role in the processing of antigen before presentation by MHC class I molecules. TAP1 and TAP2 are polymorphic and are located in the
MHC class II region. In the rat, polymorphisms of the TAP2 gene can dramatically affect the type of peptides bound by class I molecules. ${ }^{11}$ The TAP genes may therefore represent candidate susceptibility genes or useful markers for SLE and other MHC-linked diseases. To test this hypothesis, we determined the frequencies of dimorphisms and alleles of TAP2 in SLE patients who had already been characterised for HLA-DQ, DR and complement $\mathrm{C} 4 .^{5}$

TAP2A/D and TAP2C/E heterozygotes cannot be distinguished using ARMS-PCR. From the gene frequencies of these alleles, TAP2A/D heterozygotes would be expected to occur much more frequently than TAP2C/E heterozygotes. Family studies have confirmed that this is indeed the case (unpublished data). We therefore feel that it is reasonably safe to assume TAP2A/D heterozygosity in such ambiguous cases (of which there were only two in our SLE patient population).

This study clearly indicates that no dimorphism or allele of the TAP2 gene is associated with SLE. In addition, no significant associations were found in any of the clinical/immunological subgroups of SLE that were studied. These data suggest that TAP2 is not involved in determining genetic predisposition to SLE.

We have found no evidence for strong linkage disequilibrium between TAP2 and HLA-DQ/DR. Indeed, no significant linkage was observed in our patient population. We have found TAP2B to be in significant linkage disequilibrium with DR1 $(\Delta=0.056$, $\mathrm{p}=0.0002)$ in the control group used in this study. A similar but less marked situation was observed in the SLE group $(\Delta=0.025$, $\mathrm{p}=0.05$ ) although this was not statistically significant. This apparent discrepancy may be explained by the decreased frequency of DR1 in SLE compared with controls in this patient population. ${ }^{5}$ Non-significant negative linkage disequilibrium was observed between TAP2B and DR4 $(\Delta=-0.040, p=0.03)$ in our control population, but not in our SLE patient group. TAP2B exhibited non-significant negative linkage disequilibrium with DR3 in SLE patients. These findings agree with those of a previous study in which T-665 (not present in TAP2B) was found to be associated with DR4DQ8 and DR3-DQ2 haplotypes, while A-665 (present in TAP2B) was associated with DR1DQ5 haplotypes. ${ }^{12}$

The trend towards an increased frequency of TAP2D in SLE patients with circulating antibodies to U1RNP accounts for the increased frequency of threonine at amino acid position 565 in this subgroup. This finding is of particular interest due to the increased frequency of DR4 in this group of patients. ${ }^{5}$ In the control group used in this study, TAP2D is in linkage disequilibrium with DR4, suggesting that this association is probably not a chance finding. Further studies using larger numbers of patients will help to evaluate the possible association of TAP2C with anti-Ro antibodies and the lack of association of TAP2 with other subgroups of SLE. 
This lack of linkage disequilibrium between TAP2 and HLA-DQA/DR indicates that disease-predisposing haplotypes for SLE do not extend as far as TAP2, and our previously reported association of $\mathrm{DQA}^{\star} 0501$ with $\mathrm{SLE}^{5}$ did not reflect linkage with any TAP2 dimorphism/haplotype. Therefore any associations of HLA-DP alleles with $\mathrm{SLE}^{13}$ or with specific autoantibody response ${ }^{14}$ must be independent of other class II (DQ/DR) associations.

We are grateful to the Arthritis and Rheumatism Council and Lupus UK for financial support. E J Davies is funded by a Frederick Craven Moore studentship from the University of Manchester.

1 Deapen D, Escalante A, Weinrib L, et al. Revised Estimate of Twin Concordance in Systemic Lupus Erythematosus. Arthritis Rheum 1992; 35: 311-8.

2 Winchester R J, Nunez-Roldan A. Some Genetic Aspects of Systemic Lupus Erythematosus. Arthritis Rheum 1982; 25: $833-7$.

3 Arnett F C, Bias W B, Reveille J D. Genetic studies in Sjogren's syndrome and systemic lupus erythematosus. $\mathcal{F}$ Autoimmunity 1989; 2: 403-13.

4 Fronek Z, Timmerman L A, Alper C A, et al. Major histocompatibility complex genes and susceptibility to systemic lupus erythematosus. Arthritis Rheum 1990; 33: 1542-53.
5 Davies E J, Hillarby M C, Cooper R G, et al. HLA-DQ, DR and Complement C4 Variants in SLE. Brit $\mathscr{f}$ Rheumatol 1993; 32: 870-5.

6 Trowsdale J, Hanson I, Mockridge I, Beck S, Townsend A Kelly A. Sequences encoded in the class II region of the MHC related to the "ABC" superfamily of transporters. Nature 1990; 348: 741-3.

7 Bahram S, Arnold D, Bresnahan M, Strominger J L. Spies $T$. Two putative subunits of a peptide pump encoded in the human major histocompatibility complex class II region. Proc Natl Acad Sci USA 1991; 88: 10094-8.

8 Robertson M. Proteasomes in the pathway. Nature 1991; 353: 300-1.

9 Tan E M, Cohen A S, Fries J F, et al. The 1982 revised criteria for the classification of systemic lupus erythematosus. Arthritis Rheum 1982; 25: 1271-7.

10 Powis S H, Tonks S, Mockridge I, Kelly A P, Bodmer J G Trowsdale J. Alleles and haplotypes of the MHC-encoded ABC transporters TAP1 and TAP2. Immunogenetics 1993; 37: $373-80$.

11 Powis S J, Deverson E V, Coadwell W J, et al. Effect of polymorphism of an MHC-linked transporter on the peptides assembled in a class I molecule. Nature 1992; 357: $211-5$.

12 Ronningen K S, Undlien D E, Ploski R, et al. Linkage disequilibrium between TAP2 variants and HLA class II alleles; no primary association between TAP2 variants and insulin-dependent diabetes mellitus. Eur f Immunol 1993; 23: 1050-6.

13 Galeazzi M, Sebastiani G D, Passiu G, et al. HLA-DP Genotyping in Patients with Systemic Lupus Erythematosus: Correlations with Autoantibody Subsets. fRheumatol 1992; 19: 42-6.

14 Hsu K C, Hill D I, Hoffman R W. HLA-DPB1*0401 is associated with the presence of autoantibodies reactive with the U1-70kD polypeptide antigen of U1-small with the U1-70kD polypeptide antigen of U1-small nuclear ribonucleoprotein among connective
disease patients. Tissue Antigens 1992; 39: 272-5. 